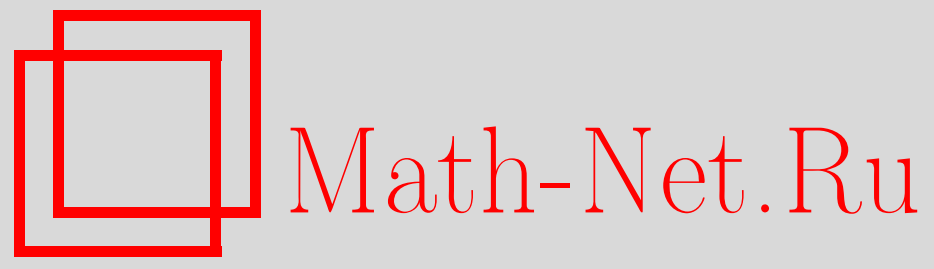

А. Г. Шоломицкий, О необходимых условиях пуассоновской сходимости мартингалов, Теория вероятн. и ее примен., 2004, том 49, выпуск 4, 813-816

DOI: https://doi.org/10.4213/tvp199

Использование Общероссийского математического портала Math-Net.Ru подразумевает, что вы прочитали и согласны с пользовательским соглашением

http://www . mathnet.ru/rus/agreement

Параметры загрузки:

IP : 35.173 .219 .12

26 апреля 2023 г., $17: 46: 17$

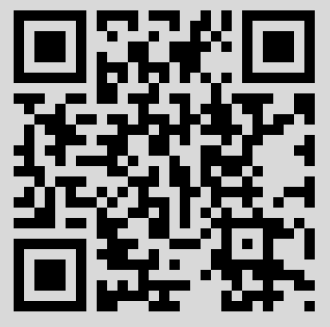


3. Розовский Л.В. Некоторые предельные теоремы для больших уклонений сумм независимых случайных величин с общей функцией распределения из области притяжения нормального закона. -- Записки научн. семин. ПОМИ, 2002, т. 294, c. 165-193.

4. Li D. L., Wang X. C., Rao M. B. Some results on convergence rates for probabilities of moderate deviations for sums of random variables. - Internat. J. Math. Math. Sci., 1992, v. 15, № 3, p. 481-498.

5. Heyde C. C. A supplement to the strong law of large numbers. - J. Appl. Probab., 1975, v. 12, p. 173-175.

6. Chen $R$. A remark on the tail probability of a distribution. - J. Multivariate Anal., 1978 , v. 8, № 2, p. 328-333.

7. Spătaru A. Precise asymptotics in Spitzer's law of large numbers. - J. Theoret. Probab., 1999, v. 12, № 3, p. 811-819.

8. Розовский Л. В. О точной асимптотике в слабом законе больших чисел для сумм независимых случайных величин с общей функцией распределения из области притяжения устойчивого закона. - Теория вероятн. и ее примен., 2003, т. 48, B. 3 , с. $589-596$.

9. Gut A., Spătaru A. Precise asymptotics in the law of the iterated logarithm. - Ann. Probab., 2000, v. 28, № 4, p. 1870-1883.

10. Феллер B. Введение в теорию вероятностей и ее приложения. Т. 2. М.: Мир, 1984, 752 c.

11. Золотарев B. М. Одномерные устойчивые распределения. М.: Наука, 1983, 304 с.

12. Розовский Л. В. Одна оценка для вероятностей больших уклонений. - Матем. заметки, 1987, т. 42, № 1, с. 145-156.

13. Петров В.В. Предельные теоремы для сумм независимых случайных величин. M.: Наука, 1987,317 с.

Поступила в редакцию 5.II. 2003

(C) $2004 \Gamma$.

$$
\begin{gathered}
\text { ШоломицКИй А. } .^{*} \\
\text { О НЕОБХОДИМЫХ УСЛОВИЯХ } \\
\text { ПУАССОНОВСКОЙ СХОДИМОСТИ МАРТИНГАЛОВ }
\end{gathered}
$$

Доказана теорема о необходимых условиях пуассоновской сходимости сумм слагаемых, являющихся мартингал-разностями. Условия теоремы обобщают классические условия сходимости сумм независимых слагаемых.

Ключевье слова и фразы: мартингал, закон Пуассона, слабая сходимость.

B [6] были получены некоторые результаты о необходимых условиях нормальной сходимости сумм мартингал-разностей. Оказывается, что тем же методом можно получить необходимые условия и для пуассоновского случая.

Ниже символом $\mathscr{L}$ обозначается закон распределения случайной величины, сходимость законов понимается в смысле слабой сходимости соответствуюших распределений, пуассоновский закон с параметром $\lambda$ обозначается $\mathscr{P}(\lambda)$. Символы $\rightarrow$ и $\stackrel{\mathrm{P}}{\longrightarrow}$

* Центральный экономико-математический институт РАН, Нахимовский пр-т, 47 , 117418 Москва, Россия; e-mail: ags@cemi.rssi.ru

1) Работа частично поддержана грантом РФФИ 03-01-00479 и грантом РАН по 6-му конкурсу-экспертизе научных проектов молодых ученых (грант № 105). 
обозначают соответственно сходимость и сходимость по вероятности при $n \rightarrow \infty$; $I(A)$ - индикатор события $A$.

Пусть при каждом $n$ на некотором вероятностном пространстве $(\Omega, \mathscr{F}, \mathbf{P})$ заданы квадратично интегрируемые случайные величины $\left\{X_{j, n}\right\}, j=1, \ldots, k_{n}<\infty$. Обозначим $S_{n}=\sum_{j=1}^{k_{n}} X_{j, n}, \mathscr{F}_{n}^{k}=\sigma\left(X_{1, n}, \ldots, X_{k, n}\right), \mathscr{F}_{n}^{0}=\{\varnothing, \Omega\}$.

Предположим, что

$$
\mathbf{E}\left(X_{j, n} \mid \mathscr{F}_{n}^{j-1}\right)=0 \quad(\text { P-п.н. })
$$

и при $n \rightarrow \infty$

$$
\sum_{j=1}^{k_{n}} \mathbf{E}\left(X_{j, n}^{2} \mid \mathscr{F}_{n}^{j-1}\right) \stackrel{\mathbf{P}}{\longrightarrow} \lambda>0
$$

Следующая теорема аналогична теореме 2, доказанной в [6] для нормального случая.

Теорема. Пусть выполнены условия (1) и (2). Пусть

$$
\begin{gathered}
\max _{j \leqslant k_{n}} \mathbf{E}\left(X_{j, n}^{2} \mid \mathscr{F}_{n}^{j-1}\right) \stackrel{\mathbf{P}}{\longrightarrow} 0, \\
\sup _{n} \sum_{j=1}^{k_{n}} \mathbf{E}\left|X_{j, n}\right|^{5} \leqslant M<\infty, \\
\sum_{j<i} \mathbf{E}\left(X_{i, n}^{3} X_{j, n}\right) \longrightarrow 0 .
\end{gathered}
$$

Тогда из пуассоновской сходимости

$$
\mathscr{L}\left(S_{n}+\lambda\right) \rightarrow \mathscr{P}(\lambda)
$$

следует, что для любого $\varepsilon>0$

$$
\sum_{j=1}^{k_{n}} \mathbf{E}\left(X_{j, n}^{2} I\left(\left|X_{j, n}-1\right| \geqslant \varepsilon\right) \mid \mathscr{F}_{n}^{j-1}\right) \longrightarrow 0 .
$$

Условие (3) представляет собой «условный» аналог свойства предельной пренебрегаемости слагаемых. Условие (4) носит технический характер. Условие (5) выполнено, например, если $\mathbf{E}\left(X_{j, n} \mid X_{i, n}\right)=0 \mathbf{P}$-п.н. для любых $i \neq j$.

Достаточность условия (6), являющегося «условной» формой классического условия, для пуассоновской сходимости $(\mathrm{P})$ следует из результатов Брауна и Иглсона [1]. Нижеследуюшее доказательство опирается на доказательство теоремы 2 из [6]. Ее доказательство в [6] широко использует теорию, развитую в [3]-[5].

Д ок а з а т л л с т в о. Без ограничения обшности полсжим $\lambda=1$. Очевидно, (Р) эквивалентно сходимости функций распределения сумм $S_{n}$ к функции распределения центрированного (с нулевым средним) пуассоновского закона, которую обозначим $\bar{\Pi}$. Пусть

$$
\zeta_{r}=\frac{1}{r !} \int_{-\infty}^{\infty} x^{r} d \bar{\Pi}(x)
$$

Введем, аналогично [6], случайную меру $K_{n}$ с функцией распределения

$$
K_{n}(x)=\sum_{j} \int_{-\infty}^{x} y^{2} d F_{j, n}\left(y \mid \mathscr{F}_{n}^{j-1}\right),
$$

где $F_{j, n}\left(y \mid \mathscr{F}_{n}^{j-1}\right)=\mathbf{P}\left(X_{j, n} \leqslant y \mid \mathscr{F}_{n}^{j-1}\right)$, а также величины $k_{n}^{(r)}=\int_{-\infty}^{\infty} x^{r} d K_{n}(x)$. Как в [6], рассмотрим асимптотически пуассоновскую последовательность усеченных случайных величин $\widetilde{S}_{n}=S_{n} I\left(\left|S_{n}\right| \leqslant C_{n}\right), C_{n} \rightarrow \infty$, такую, что для некоторого числа $M_{1}<\infty$

$$
\mathbf{E}\left|\widetilde{S}_{n}\right|^{5} \leqslant M_{1}
$$


и, следовательно, все моменты порядка $m \leqslant 4$ сходятся к моментам предельного распределения:

$$
(m !)^{-1} \mathbf{E} \widetilde{S}_{n}^{m} \rightarrow \zeta_{m}
$$

Обозначим через $\mu_{n r}$ коэффициенты тейлоровского разложения функции

$$
\mathbf{E} \frac{e^{i t \widetilde{S}_{n}}}{\exp \left\{\int\left(e^{i t x}-1-i t x+(t x)^{2} / 2\right) d K_{n}(x) / x^{2}\right\}} .
$$

Как показано в [6] (см. соотношение $(20)$, с. 506), для $m \leqslant 4$

$$
\left|\mu_{n m}-\zeta_{m}\right| \rightarrow 0 \text {. }
$$

Для $m=3$, собирая члены с $(i t)^{3}$, получаем из $(9)$

$$
\frac{(i t)^{3}}{3 !} \mathbf{E} \widetilde{S}_{n}^{3}-\frac{(i t)^{3}}{3 !} \mathbf{E} k_{n}^{(1)} \longrightarrow 0 .
$$

Так как $\zeta_{3}=\frac{1}{3 !}$, то в силу (8) $\mathbf{E} \widetilde{S}_{n}^{3} \rightarrow 1$, откуда $\mathbf{E} k_{n}^{(1)} \longrightarrow 1$.

Для $m=4$, собирая члены разложения с $(i t)^{4}$, имеем из $(9)$

$$
\frac{(i t)^{4}}{4 !} \mathbf{E} \widetilde{S}_{n}^{4}-\frac{(i t)^{4}}{3 !} \mathbf{E} \widetilde{S}_{n} k_{n}^{(1)}-\frac{(i t)^{4}}{4 !} \mathbf{E} k_{n}^{(2)}-\frac{t^{4}}{8} \longrightarrow 0 .
$$

Как показано в [6] (с. 507), $\mathbf{E} \widetilde{S}_{n} k_{n}^{(1)} \rightarrow 0$, поэтому второе слагаемое в (10) стремится к нулю. Так как в силу (8) $\mathbf{E} \widetilde{S}_{n}^{4} \rightarrow 4 ! \zeta_{4}=4$, то из (10) следует, что

$$
\mathbf{E} k_{n}^{(2)} \rightarrow 4 !\left(\frac{4}{4 !}-\frac{1}{8}\right) \rightarrow 1
$$

Теперь запишем неравенство

$$
\left(\mathbf{E} k_{n}^{(1)}\right)^{2} \leqslant \mathbf{E}\left(k_{n}^{(1)}\right)^{2} \leqslant \mathbf{E} k_{n}^{(2)}
$$

в котором левая и правая части, по доказанному, стремятся к единице. Поэтому средняя часть также стремится к единице. Так как она представляет собой второй момент случайной величины $k_{n}^{(1)}$, математическое ожидание которой, как было показано, также сходится к 1 , то дисперсия этой величины стремится к 0 и, следовательно,

$$
k_{n}^{(1)} \stackrel{\mathbf{P}}{\longrightarrow} 1 .
$$

Рассмотрим теперь случайную величину $k_{n}^{(2)}$. Так как $k_{n}^{(2)} \geqslant\left(k_{n}^{(1)}\right)^{2} \stackrel{\mathbf{P}}{\longrightarrow} 1$ и, с другой стороны, $\mathbf{E} k_{n}^{(2)} \rightarrow 1$, имеем $k_{n}^{(2)} \stackrel{\mathbf{P}}{\longrightarrow} 1$. Таким образом, два первых момента случайной меры $K_{n}$ стремятся к 1 по вероятности. Поэтому

$$
\begin{gathered}
\sum_{j=1}^{k_{n}} \mathbf{E}\left(X_{j, n}^{2} I\left(\left|X_{j, n}-1\right| \geqslant \varepsilon\right) \mid \mathscr{F}_{n}^{j-1}\right)=\int_{|x-1| \geqslant \varepsilon} d K_{n} \leqslant \frac{1}{\varepsilon^{2}} \int(x-1)^{2} d K_{n}(x) \\
=\frac{1}{\varepsilon^{2}}\left\{k_{n}^{(2)}-2 k_{n}^{(1)}+\int_{\mathbf{R}} d K_{n}\right\}=\frac{1}{\varepsilon^{2}}\left\{k_{n}^{(2)}-2 k_{n}^{(1)}+\sum_{j} \sigma_{j, n}^{2}\right\} \stackrel{\mathbf{P}}{\longrightarrow} 0,
\end{gathered}
$$

что и треоовалось доказать.

\section{СПИСОК ЛИТЕРАТУРЫ}

1. Brown B., Eagleson G.K. Martingale convergence to infinitely divisible laws with finite variance. - Trans. Amer. Math. Soc., 1971, v. 162, p. 449-453.

2. Brown B. Martingale central limit theorems. -- Ann. Math. Statist., 1971, v. 42, p. $59-66$.

3. Жикод Ж., Ширяев А. Н. Предельные теоремы для случайных процессов. Т. 1, 2. М.: Наука, 1994, 544 с., 368 с. 
4. Липцер P.Ш., Ширяев $A . H$. Функциональная центральная предельная теорема для семимартингалов. - Теория вероятн. и ее примен., 1980, т. 25, в. 4, с. $683-$ 703.

5. Липиер Р.Ш., Ширяев А. Н. Теория мартингалов. - М.: Наука, 1986, 512 с.

6. Шоломиикий А. Г. О необходимых условиях нормальной сходимости для мартингалов. - Теория вероятн. и ее примен., 1998 , т. 43 , в. 3, с. 490-508.

Поступила в редакцию

14.XI.2001

(c) $2004 \Gamma$.

NOWAK E.*

\title{
ABSOLUTE CONTINUITY BETWEEN A GIBBS MEASURE AND ITS TRANSLATE ${ }^{1)}$
}

\begin{abstract}
Наша первая цель - получить оценку для расстояния по вариации между мерой Гиббса на пространстве $\mathbf{R}^{\mathbf{Z}^{d}}$ и ее сдвигом на вектор из этого пространства. Это может быть сделано, если контролировать взаимозависьимость между спинами в различных позициях, т.е. налагать некоторые ограничения на соответствующий потенциал. Мы можем затем заключить, в точно описанных случаях, что исходная мера и ее сдвиг эквивалентны.
\end{abstract}

Ключевые слова и фразы: случайные поля, расстояние по вариации, меры Гиббса, эквивалентность мер.

1. Introduction. Given a measure $\mu$ on a measurable space $(\Omega, \mathscr{F})$ and $b$ an element of $\Omega$, we define its translate by $\mu^{(b)}(B)=\mu(B-b)$ for any $B$ in $\mathscr{F}$. The question of distinguishing $\mu$ and $\mu^{(b)}$ has been introduced by Shepp [18] in the following case: let $X=\left\{X_{k}, k \in \mathbf{N}\right\}$ be a sequence of independent and identically distributed (i.i.d.) random variables whose Fisher information is finite, and let $\mu$ be the measure induced by $X$. Then Shepp has proven that $\mu$ and $\mu^{(b)}$ are equivalent if and only if $b$ belongs to $\ell^{2}(\mathbf{N})$. Since, the problem of distinguishing a sequence of i.i.d. random variables from a translate of itself has been widely studied (see, for example, [8] or [13j).

The aim of this paper is to remove this independence condition. The normal case is also well known: see Feldman [5] Rozanov [14], [15], Skorokhod and Yadrenko [21], Skorokhod [20, Chapter 3], Ibragimov and Rozanov [7, Chapter 3], and Lifshits [10, Section 9] for general questions of absolute continuity and singularity of Gaussian measures. Differentiability of measures corresponding to Markov processes has also been studied by Skorokhod [19, Chapter 4] and Sato [17], and Noquet [11] has recently given results for homogeneous Markov chains.

Gaussian and Markov random fields can be considered, in some cases, as Gibbs random fields: first results on this topic have been given by Rozanov [16] followed by Dobrushin [3], Künsch [9] and Georgii [6, Chapters 10-13]. We focus here on Gibbs measures on $\mathbf{R}^{\mathbf{Z}^{d}}$, using a method based on the distance in total variation. At last, let us point out that the study of the distance in total variation between the law of an i.i.d. random variables sequence and its translate has led Davydov and Lifshits [1] to a local invariance principle

* Laboratoire de Matehématiques Paul Painlevé, U.M.R. CNRS 8524, Université des Sciences et Technologies de Lille, U.F.R. de Mathématiques, 59655 Villeneuve d'Ascq Cedex, France; e-mail: e.nowak@isa-lille.fr

1) Research was partially supported by the Institut Supérieur d'Agriculture (Lille, France). 